\title{
THE GENERALIZED CHAZY EQUATION AND SCHWARZIAN TRIANGLE FUNCTIONS*
}

\author{
M. J. ABLOWITZ ${ }^{\dagger}$, S. CHAKRAVARTY ${ }^{\ddagger}$, AND R. HALBURD ${ }^{\S}$
}

\begin{abstract}
An integrable third-order system of Darboux-Halphen type that depends on three arbitrary parameters $\alpha, \beta$, and $\gamma$ is considered. The general solution of this system is given in terms of Schwarzian triangle functions associated with circular triangles with angles $\alpha \pi, \beta \pi$, and $\gamma \pi$. It is shown that when the three parameters $\alpha, \beta$, and $\gamma$ are equal or when two of the parameters are $1 / 3$ the Darboux-Halphen system reduces to the generalized Chazy equation which is a classically known third-order scalar polynomial ordinary differential equation. This gives rise to representations of the solution of the generalized Chazy equation in terms of Schwarzian functions that differ from that given by Chazy. These results are then used to derive relations between various Schwarzian triangle functions.
\end{abstract}

1. Introduction. In $[1,2]$ the authors obtained the system

$$
\begin{aligned}
& \dot{\omega}_{1}=\omega_{2} \omega_{3}-\omega_{1}\left(\omega_{2}+\omega_{3}\right)+a, \\
& \dot{\omega}_{2}=\omega_{3} \omega_{1}-\omega_{2}\left(\omega_{3}+\omega_{1}\right)+a, \\
& \dot{\omega}_{3}=\omega_{1} \omega_{2}-\omega_{3}\left(\omega_{1}+\omega_{2}\right)+a,
\end{aligned}
$$

where

$$
a=\alpha^{2}\left(\omega_{1}-\omega_{2}\right)\left(\omega_{3}-\omega_{1}\right)+\beta^{2}\left(\omega_{2}-\omega_{3}\right)\left(\omega_{1}-\omega_{2}\right)+\gamma^{2}\left(\omega_{3}-\omega_{1}\right)\left(\omega_{2}-\omega_{3}\right),
$$

and $\alpha, \beta$, and $\gamma$ are constants, as a special case of the Darboux-Halphen system DH-IX. Without loss of generality we choose $\alpha, \beta$, and $\gamma$ in (2) to have non-negative real parts. The general solution of the system (1-2) is given in terms of Schwarzian triangle functions which are associated with circular triangular regions with angles $\alpha \pi$, $\beta \pi$, and $\gamma \pi$ (see $[1,2]$ and below). The case $a=0$ is the classical Darboux-Halphen system which appeared in Darboux's analysis of triply orthogonal surfaces [3] and was later solved by Halphen [4]. The classical Darboux-Halphen system, which is also equivalent to the vacuum Einstein equations for Riemannian self-dual Bianchi-IX metrics $[5,6]$, is equivalent to the classical Chazy equation [7]

$$
\frac{d^{3} y}{d t^{3}}=2 y \frac{d^{2} y}{d t^{2}}-3\left(\frac{d y}{d t}\right)^{2},
$$

where

$$
y:=-2\left(\omega_{1}+\omega_{2}+\omega_{3}\right)
$$

Halphen also studied and solved equations (1-2) [8]. For special choices of $(\alpha, \beta, \gamma)$, solutions of equations (1-2) have been obtained in terms of automorphic

${ }^{*}$ Received January 21, 1999; accepted for publication April 6, 1999. The work reported in this paper was partially supported by NSF grant number D.IS - 9703850 .

$\dagger$ Department of Applied Mathematics, University of Colorado at Boulder, Boulder, CO, 80309526, USA (markjab@newton.colorado.edu).

$\ddagger$ Department of Applied Mathematics, University of Colorado at Boulder, Boulder, CO, 80309526 , USA (chuck@newton.colorado.edu). The research of this author is partially supported by an Australian Research Council Grant, no. A69803721.

$\S$ Department of Applied Mathematics, University of Colorado at Boulder, Boulder, CO, 80309526, USA (rod@newton.colorado.edu). 
forms [9, 10]. In [2] the authors used Schwarzian triangle functions to show that $\alpha=\beta=\gamma=2 / n$ or $\alpha=\beta=1 / 3, \gamma=2 / n$, et cyc., where $n$ is a constant, are the only choices of parameter for which $y$ defined by (4) satisfies an equation of polynomial type. In all cases this equation is

$$
\frac{d^{3} y}{d t^{3}}-2 y \frac{d^{2} y}{d t^{2}}+3\left(\frac{d y}{d t}\right)^{2}=\frac{4}{36-n^{2}}\left(6 \frac{d y}{d t}-y^{2}\right)^{2},
$$

which was also studied by Chazy $[11,7,12]$ and is often referred to as the generalized Chazy equation. In the next section we will show this result directly. The case $n=\infty$ in equation (5) corresponds to equation (3), the classical Chazy equation.

Through our solution of the system (1-2) in terms of various Schwarzian triangle functions, we also obtain a number of representations (via equation 4 ) of the solution of the generalized Chazy equation (5) in terms of different Schwarzian triangle functions. Chazy also (implicitly) solved equation (5) in terms of Schwarzian triangle functions which are different from any of the representations obtained here. We show how these representations can be used to obtain a number of identities between the Schwarzian functions (hauptmodules) of different triangular groups.

The reduction of the SDYM equations to DH-IX induces a corresponding reduction from the associated linear problem of SDYM [13] to a linear problem for DH-IX $[14,15]$. This linear problem is monodromy-evolving in contrast to the isomonodromy problems associated with the Painlevé equations. The general solution of the system (1-2) is densely branched for generic $\alpha, \beta, \gamma$ and so does not possess the Painlevé property, which is closely associated with integrability (see [16, 17, 18, 19, 20, 21]).

2. The Generalized Chazy Equation. In this section we will find all choices of the parameters $\alpha, \beta$, and $\gamma$ in the Darboux-Halphen system (1-2) for which $y$ defined by equation (4) satisfies the generalized Chazy equation (5). Without loss of generality we take $\Re e(n) \geq 0$. Using the system (1-2) we obtain

$$
\begin{aligned}
\frac{d y}{d t}= & 2\left(\omega_{2} \omega_{3}+\omega_{3} \omega_{1}+\omega_{1} \omega_{2}\right)-6 a, \\
\frac{d^{2} y}{d t^{2}}= & -12 \omega_{1} \omega_{2} \omega_{3}+4\left(\omega_{1}+\omega_{2}+\omega_{3}\right) a-6 \frac{d a}{d t}, \\
\frac{d^{3} y}{d t^{3}}= & -12\left(\omega_{2}^{2} \omega_{3}^{2}+\omega_{3}^{2} \omega_{1}^{2}+\omega_{1}^{2} \omega_{2}^{2}\right)+24\left(\omega_{1}+\omega_{2}+\omega_{3}\right) \omega_{1} \omega_{2} \omega_{3} \\
& -16\left(\omega_{2} \omega_{3}+\omega_{3} \omega_{1}+\omega_{1} \omega_{2}\right) a+12 a^{2}+4\left(\omega_{1}+\omega_{2}+\omega_{3}\right) \frac{d a}{d t}-6 \frac{d^{2} a}{d t^{2}},
\end{aligned}
$$

where

$$
\begin{aligned}
\frac{d a}{d t}= & -2\left\{\alpha^{2}\left(\omega_{2}+\omega_{3}\right)\left(\omega_{3}-\omega_{1}\right)\left(\omega_{1}-\omega_{2}\right)+\beta^{2}\left(\omega_{3}+\omega_{1}\right)\left(\omega_{1}-\omega_{2}\right)\left(\omega_{2}-\omega_{3}\right)\right. \\
& \left.+\gamma^{2}\left(\omega_{1}+\omega_{2}\right)\left(\omega_{2}-\omega_{3}\right)\left(\omega_{3}-\omega_{1}\right)\right\},
\end{aligned}
$$

and

$$
\begin{aligned}
\frac{d^{2} a}{d t^{2}}= & 4\left\{\alpha^{2}\left(\omega_{3}-\omega_{1}\right)\left(\omega_{1}-\omega_{2}\right)\left(\omega_{2}^{2}+3 \omega_{2} \omega_{3}+\omega_{3}^{2}-a\right)\right. \\
& +\beta^{2}\left(\omega_{1}-\omega_{2}\right)\left(\omega_{2}-\omega_{3}\right)\left(\omega_{3}^{2}+3 \omega_{3} \omega_{1}+\omega_{1}^{2}-a\right) \\
& \left.+\gamma^{2}\left(\omega_{2}-\omega_{3}\right)\left(\omega_{3}-\omega_{1}\right)\left(\omega_{1}^{2}+3 \omega_{1} \omega_{2}+\omega_{2}^{2}-a\right)\right\} .
\end{aligned}
$$


By substitution of the above results, the generalized Chazy equation (5) now has the form

$$
\begin{aligned}
& \left\{16\left(\omega_{1}+\omega_{2}+\omega_{3}\right)^{2}-88\left(\omega_{2} \omega_{3}+\omega_{3} \omega_{1}+\omega_{1} \omega_{2}\right)\right\} a+120 a^{2} \\
& -20\left(\omega_{1}+\omega_{2}+\omega_{3}\right) \frac{d a}{d t}-6 \frac{d^{2} a}{d t^{2}} \\
= & \frac{64}{36-n^{2}}\left\{\left(1-9 \alpha^{2}\right)\left(\omega_{1}-\omega_{2}\right)\left(\omega_{3}-\omega_{1}\right)+\left(1-9 \beta^{2}\right)\left(\omega_{2}-\omega_{3}\right)\left(\omega_{1}-\omega_{2}\right)\right. \\
& \left.+\left(1-9 \gamma^{2}\right)\left(\omega_{3}-\omega_{1}\right)\left(\omega_{2}-\omega_{3}\right)\right\}^{2} .
\end{aligned}
$$

Equation (6) must be satisfied for all solutions $\omega_{1}, \omega_{2}, \omega_{3}$ of the system (1-2). In particular, we consider the three solutions with initial conditions $\left(\omega_{1}(0), \omega_{2}(0), \omega_{3}(0)\right)$ given by $(1,0,0),(0,1,0)$, and $(0,0,1)$ respectively. For these three solutions, equation (6) at $t=0$ yields the following three equations

$$
\left(1-9 \alpha^{2}\right)\left(n^{2} \alpha^{2}-4\right)=0, \quad\left(1-9 \beta^{2}\right)\left(n^{2} \beta^{2}-4\right)=0, \quad\left(1-9 \gamma^{2}\right)\left(n^{2} \gamma^{2}-4\right)=0 .
$$

If none of the parameters $\alpha, \beta, \gamma$ are $1 / 3$ then $\alpha=\beta=\gamma=2 / n$ and equation (6) is identically satisfied. If exactly one of the parameters is $1 / 3$ (say $\alpha=1 / 3$ ) then $\beta=\gamma=2 / n$ and the right side of equation (6) is independent of $\omega_{1}$ but the left side is not. So we cannot have exactly one of the parameters equal to $1 / 3$. If $\alpha=\beta=1 / 3$ and $\gamma \neq 1 / 3$ then $\gamma=2 / n$ and equation (6) is identically satisfied. Since we can cyclically permute $(\alpha, \beta, \gamma)$, we see that the only choices of $(\alpha, \beta, \gamma)$ for which $y$ defined by (4) and (1-2) are

$$
(2 / n, 2 / n, 2 / n), \quad(1 / 3,1 / 3,2 / n), \quad(2 / n, 1 / 3,1 / 3), \quad(1 / 3,2 / n, 1 / 3) .
$$

Alternatively, one could substitute the expressions for $a, \frac{d a}{d t}$ and $\frac{d^{2} a}{d t^{2}}$ into equation (6) and equate the coefficients of the resulting polynomial in $\omega_{1}, \omega_{2}$, and $\omega_{3}$ to zero and solve for $\alpha, \beta$, and $\gamma$.

3. Schwarzian Triangle Functions. In $[1,2]$ it was shown that the general solution of the Darboux-Halphen system (1-2) is given by

$$
\begin{aligned}
\omega_{1} & =-\frac{1}{2} \frac{d}{d t} \ln \frac{\dot{s}}{s(s-1)}, \\
\omega_{2} & =-\frac{1}{2} \frac{d}{d t} \ln \frac{\dot{s}}{s-1}, \\
\omega_{3} & =-\frac{1}{2} \frac{d}{d t} \ln \frac{\dot{s}}{s}
\end{aligned}
$$

where $s$ is the general solution of the Schwarzian equation

$$
\{s, t\}+\frac{\dot{s}^{2}}{2} V(s)=0
$$

and

$$
\{s, t\}:=\frac{d}{d t}\left(\frac{\ddot{s}}{\dot{s}}\right)-\frac{1}{2}\left(\frac{\ddot{s}}{\dot{s}}\right)^{2}
$$


is the Schwarzian derivative and $V$ is given by

$$
V(s)=\frac{1-\beta^{2}}{s^{2}}+\frac{1-\gamma^{2}}{(s-1)^{2}}+\frac{\beta^{2}+\gamma^{2}-\alpha^{2}-1}{s(s-1)} .
$$

In order to make the parameter dependence explicit, we will sometimes write a solution of equation (9) as $s(\alpha, \beta, \gamma ; t)$. The general solution of the Schwarzian equation (9) is given implicitly by

$$
t(s)=\frac{\chi_{2}(s)}{\chi_{1}(s)}
$$

where $\chi_{1}(s)$ and $\chi_{2}(s)$ are two independent solutions of the hypergeometric equation

$$
s(1-s) \frac{d^{2} \chi}{d s^{2}}+[c-(a+b+1) s] \frac{d \chi}{d s}-a b \chi=0,
$$

where $a=(1+\alpha-\beta-\gamma) / 2, b=(1-\alpha-\beta-\gamma) / 2$, and $c=1-\beta$ (see, e.g. $[22,23]$ ).

From equations (4), (8) and the results of the last section, we see that $y$ can be represented in terms of any of the Schwarzian triangle functions $s(2 / n, 2 / n, 2 / n ; t)$, $s(1 / 3,1 / 3,2 / n ; t), s(2 / n, 1 / 3,1 / 3 ; t)$, and $s(1 / 3,2 / n, 1 / 3 ; t)$ by

$$
y(t)=\frac{1}{2} \frac{d}{d t} \ln \frac{\dot{s}^{6}}{s^{4}(s-1)^{4}} .
$$

On the other hand, Chazy [12] analyzed equation (5) and showed that its solution is related to the Schwarz function $J=s(1 / n, 1 / 3,1 / 2)$. In terms of $J$ and its derivatives, the general solution of equation (5) is given by

$$
y(t)=\frac{1}{2} \frac{d}{d t} \ln \frac{\dot{J}^{6}}{J^{4}(J-1)^{3}}
$$

(see, for example, [23] page 195). The function $\mathrm{J}$, and hence $y$, is single-valued if $n$ is an integer greater than one.

Equating the expressions (13) and (14), we obtain

$$
\frac{\dot{J}^{6}}{J^{4}(J-1)^{3}}=\kappa \frac{\dot{s}^{6}}{s^{4}(s-1)^{4}},
$$

where $\kappa$ is a constant of integration. On taking the logarithmic derivative of equation (15), solving for the expression $\frac{d}{d t} \ln \dot{J}$ and forming the Schwarzian derivative $\{J, t\}$, we find

$$
\begin{aligned}
& \{J, t\}+\frac{\dot{J}^{2}}{2}\left(\frac{8 / 9}{J^{2}}+\frac{3 / 4}{(J-1)^{2}}-\frac{1 / 6}{J(J-1)}\right) \\
= & \{s, t\}+\frac{\dot{s}^{2}}{2}\left(\frac{8 / 9}{s^{2}}+\frac{8 / 9}{(s-1)^{2}}-\frac{8 / 9}{s(s-1)}\right) .
\end{aligned}
$$

Using the fact that $\mathrm{J}$ solves equation (9) with (10) for $\alpha=1 / n, \beta=1 / 3, \gamma=1 / 2$ and $s$ solves the same equation with $(\alpha, \beta, \gamma)$ equal to one of the choices in $(7)$, we see that equation (16) becomes

$$
J=K \frac{\left\{\left(\alpha^{2}-1 / 9\right) s^{2}+\left(\gamma^{2}-\beta^{2}-\alpha^{2}+1 / 9\right) s+\left(\beta^{2}-1 / 9\right)\right\}^{3}}{s^{2}(s-1)^{2}},
$$


whère $K=\kappa^{-1}\left(n^{-2}-1 / 36\right)^{-3}$ and we have used equation (15). Here a solution of equation (9) is defined up to a fractional linear transformation $t \mapsto(A t+B) /(C t+D)$, $A D-B C=1$. For each of the cases (7), the value of $\kappa$ can be determined by substituting $\mathrm{J}$ given by equation (17) into the Schwarzian equation (9) with $\alpha=1 / n$, $\beta=1 / 3, \gamma=1 / 2$ and using the fact that $s$ also solves a Schwarzian equation. The resulting transformations are summarized in Table 1.

TABLE 1

Transformations between Sch, .zian Triangle Functions

\begin{tabular}{|l|l|c|}
\hline$s=s(\alpha, \beta, \gamma ; t)$ & $J=s(1 / n, 1 / 3,1 / 2 ; t)$ & $\kappa$ \\
\hline$s(2 / n, 2 / n, 2 / n ; t)$ & $J=\frac{4}{27} \frac{\left(s^{2}-s+1\right)^{3}}{s^{2}(s-1)^{2}}$ & 432 \\
\hline$s(1 / 3,1 / 3,2 / n ; t)$ & $J=-\frac{4 s}{(s-1)^{2}}$ & -16 \\
\hline$s(1 / 3,2 / n, 1 / 3 ; t)$ & $J=4 \frac{(s-1)}{s^{2}}$ & -16 \\
\hline$s(2 / n, 1 / 3,1 / 3 ; t)$ & $J=-4 s(s-1)$ & -16 \\
\hline
\end{tabular}

Note that the last three transformations in Table 1 are related by the fractional linear transformations $s \mapsto 1-s$ (between the second and third transformations) and $s \mapsto 1 / s$ (between the third and fourth). These fractional linear transformations cyclically permute the angles $(\alpha, \beta, \gamma)=(1 / 3,1 / 3,2 / n)$ in equation (9) with $V$ given by (10). However the first relation between $J$ and $s$ is "symmetric" since it is invariant under these transformations. In the special case $n=\infty$, this is a classically known result [24] between the the hauptmodule $J(0,1 / 3,1 / 2 ; t)$ of the full modular group $\Gamma$ and the hauptmodule $s(0,0,0 ; t)$ of the subgroup $\Gamma(2)$. Recall that the case $n=\infty$ corresponds to the Chazy equation (3) which is equivalent to the classical DarbouxHalphen system (equation (1) with $a=0$ ). In this case Takhtajan [25] has also written the solution of the Chazy equation in terms of $s(1 / 3,1 / 3,0 ; t)$. We note that the relation between $J(0,1 / 3,1 / 2 ; t)$ and $s(1 / 3,1 / 3,0 ; t)$ is contained in Table 1 (with $n=\infty)$.

\section{REFERENCES}

[1] M.J. Ablowitz, S. Chakravarty, and R. Halburd, Darboux-Halphen systems and the singularity structure of its solutions, in Proc. Fourth Int. Conf. on Mathematical and Numerical Aspects of Wave Propagation, J. A. DeSanto, ed., SIAM, Philadelphia, 1998, pp. 408-412.

[2] .M. J. Ablowitz, S. Chakravarty, AND R. Halburd, The generalized chazy equation from the self-duality equations, Stud. Appl. Math., 1999, to appear.

[3] G. Darboux, Sur la théorie des coordonnées curvilignes et les systémes orthogonaux, Ann. Ec. Normale Supér., 7 (1878), pp. 101-150.

[4] G. Halphen, Sur un système d'équations différentielles, C. R. Acad. Sci. Paris, 92 (1881), pp. 1101-1103.

[5] G. W. GibBons And C. N. Pope, The positive action conjecture and asymptotically Euclidean metrics in quantum gravity, Commun. Math. Phys., 66 (1979), pp. 267-290.

[6] N. J. Hitchin, Monopoles, Minimal Surfaces and Algebraic Curves, Séminaire de Mathématiques Supérieuries, 105, Presses de l'Université de Montréal, Montreal, 1987.

[7] J. CHAzY, Sur les équations différentielles dont l'intégrale générale possède une coupure essentielle mobile, C.R. Acad. Sc. Paris, 150 (1910), pp. 456-458. 
[8] G. Halphen, Sur certains systèmes d'équations différentielles, C. R. Acad. Sci. Paris, 92 (1881), pp. 1404-1406.

[9] Y. OHYAмA, Systems of nonlinear differential equations related to second order linear equations, Osaka J. Math, 33 (1996), pp. 927-949.

[10] J. HARNAD AND J. MCKAY, Modular solutions to equations of generalized Halphen type, SolvInt/9804006, 1998.

[11] J. CHAZY, Sur les équations différentielles dont l'intégrale générale est uniforme et admet des singularities essentielles mobiles, C.R. Acad. Sc. Paris, 149 (1909), pp. 563-565.

[12] J. CHAZY, Sur les équations différentielles du troisième et d'ordre supérieur dont l'intégrale générale à ses points critiques fixés, Acta Math., 34 (1911), pp. 317-385.

[13] A. A. BELAVIN AND V. E. ZAKHAROV, Yang-Mills equations as inverse scattering problems, Phys. Lett. B, 73 (1978), pp. 53-57.

[14] S. Chakravarty And M. J. Ablowitz, Integrability, monodromy, evolving deformations, and self-dual Bianchi IX systems, Phys. Rev. Lett., 76 (1996), pp. 857-860.

[15] M. J. Ablowitz, S. Chakravarty, and R. Halburd, Darboux-Halphen type equations and evolving monodromy problems, U. of Colorado, Applied Math. APPM Report no. 374, May 1998.

[16] M. J. Ablowitz And H. Segur, Exact linearization of a Painlevé transcendent, Phys. Rev. Lett., 38 (1977), pp. 1103-1106.

[17] M. J. Ablowitz, A. Ramani, AND H. Segur, Nonlinear evolution equations and ordinary differential equations of Painlevé type, Lett. Nouvo Cim., 23 (1978), pp. 333-338.

[18] M. J. Ablowitz, A. Ramani, AND H. Segur, A connection between nonlinear evolution equations and ordinary differential equations of P-type. I and II, J. Math. Phys., 21 (1980), pp. 715-721 and pp. 1006-1015.

[19] M. J. Ablowitz AND P. A. Clarkson, Solitons, Nonlinear Evolution Equations and Inverse Scattering, Lond. Math. Soc. Lecture Note Series, 149, Cambridge University Press, Cambridge, 1991.

[20] M. D. Kruskal and P.A. Clarkson, The Painlevé-Kowalevski and poly-Painlevé tests for integrability, Stud. Appl. Math., 86 (1992), pp. 87-165.

[21] M. D. KRUSKal, N. Joshi, AND R. HAlbuRd, Analytic and asymptotic methods for nonlinear singularity analysis: a review and extensions of tests for the Painlevé property, in Integrability of Nonlinear Systems, (Pondicherry, 1996), Lecture Notes in Phys., 495, B. Grammaticos and K. Tamizhmani, ed., Springer, Berlin, 1997, pp. 171-205.

[22] Z. Nehari, Conformal Mapping. McGraw-Hill, New York, 1952.

[23] M. J. Ablowitz AND A. S. Fokas, Complex Variables: Introduction and Applications, CUP, Cambridge, 1997.

[24] L. R. Ford, Automorphic Forms, Chelsea, New York, 1929.

[25] L. A. TAKHTAJAN, A simple example of modular forms as tau-functions for integrable equations, Theoret. and Math. Phys., 93 (1992), pp. 1308-1317. 